\title{
Efficacy of Intralesional and Oral Dutasteride in the Treatment of Androgenetic Alopecia: A Systematic Review
}

\author{
Maira Elizabeth Herz-Ruelas ${ }^{a}$ Neri Alejandro Álvarez-Villalobos ${ }^{b}$ \\ Juan Manuel Millán-Alanís ${ }^{b}$ Humberto de León-Gutiérrez ${ }^{c}$ \\ Sonia Sofía Ocampo-Garza ${ }^{a}$ Minerva Gómez-Flores ${ }^{a}$ Ramón Grimalt ${ }^{d}$ \\ aServicio de Dermatología, Hospital Universitario “Dr. José Eleuterio González”, U.A.N.L, Monterrey, Mexico; \\ bPlataforma INVEST Medicina UANL-KER Unit Mayo Clinic (KER Unit Mexico), Universidad Autónoma de Nuevo León, \\ Monterrey, Mexico; 'School of Medicine, Hospital Universitario “Dr. José Eleuterio González", U.A.N.L, Monterrey, \\ Mexico; dFacultat de Medicina i Ciències de la Salut, Universitat Internacional de Catalunya, Barcelona, Spain
}

\section{Keywords \\ Androgenetic alopecia · 5-a-reductase inhibitors · \\ Dutasteride $\cdot$ Mesotherapy $\cdot$ Intralesional}

\begin{abstract}
Androgenetic alopecia is the most common cause of hair loss [Br J Dermatol. 2011 Jan;164(1):5-15]. Finasteride and minoxidil are the only approved treatments [J Am Acad Dermatol. 2008 Oct;59(4):547-8 and J Eur Acad Dermatology Venereol. 2018 Jan;32(1):11-22]. Dutasteride is more potent than finasteride due to its ability to inhibit both 5-a-reductase type I and II [OurDermatolOnline. 2017 Sep;9(1):75-9] though its adverse effects and long half-life contribute to the reluctance on its oral use. Mesotherapy could be a feasible alternative to avoid systemic exposure and side effects [ $J$ PanArab League Dermatologist. 2009 Feb;20(1):137-45]. We aim to perform a systematic review to analyze scientific literature with the purpose of comparing efficacy and adverse effects of both administration routes. Five clinical trials using oral route and 3 intralesional in comparison with placebo met criteria for inclusion. Regarding intralesional dutasteride, only one study [Clin Dermatol. 2001 Mar;19(2):149-54] reported the mean change in hair count. Although both inter-
\end{abstract}

ventions favor over placebo, there are not enough data to reliably compare outcomes obtained between both routes. Mean increase in hair count observed with oral dutasteride was higher (MD: 15.92 hairs [95\% Cl: 9.87-21.96]; $p=$ $<0.00001 ; R^{2}=90 \%$ ) compared to intralesional dutasteride in Abdallah's study (MD: 7.90 hairs [95\% Cl: 7.14-8.66]; $p=$ $<0.00001)$. Future studies are required to assess the therapeutic efficacy of both treatment routes, including head-tohead treatments before well-supported conclusions can be established.

c) 2020 S. Karger AG, Basel

\section{Introduction}

Androgenetic alopecia (AGA) is the most common cause of hair loss affecting both men and women [1]. By the age of 70 or beyond, $80 \%$ of Caucasian men and up to $40 \%$ of women have signs of AGA $[2,3]$. It is a multifactorial and polygenetic condition involving diverse factors such as endocrine abnormalities, circulating androgens, and microinflammation [4-6], with significant impairment on quality of life often causing psychological distress $[7,8]$. The main pathological mechanism of AGA karger@karger.com

www.karger.com/sad

Karger ${ }^{\prime}=$
(C) 2020 S. Karger AG, Basel

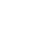


fundamentally involves the local and systemic conversion of testosterone to dihydrotestosterone (DHT) by the enzyme 5 - $\alpha$-reductase ( $5 \mathrm{aR}$ ).

There are 3 isoenzymes of 5 aR: types I, II, and III [9]. Type I is mainly present in hair follicles and sweat and sebaceous glands [10, 11], whereas type II is predominantly found in the male genitalia, including the prostate, but also in the inner root sheath of hair follicles. Type III has been found throughout the dermis and epidermis, as well as in the mammary gland and brain, but its function has yet to be elucidated [12]. DHT binds to the androgen receptor, and the hormone-receptor complex then activates the genes responsible for the gradual miniaturization of large, terminal hair follicles. Over successive hair cycles, the duration of anagen shortens and matrix size decreases, thus resulting in vellus hairs, which are the feature of AGA $[12,13]$.

Various treatments have been attempted for AGA, such as topical minoxidil, topical or systemic use of $5 \mathrm{aR}$ inhibitors, antiandrogens, low-light laser therapy, and platelet-rich plasma. Also, evidence-based guidelines for the treatment of AGA in women and men are available, but there are only 2 FDA-approved therapies for this purpose: finasteride, a type II $5 \mathrm{aR}$ inhibitor, and minoxidil, a vasodilator $[14,15]$.

Dutasteride, a 2nd-generation inhibitor of $5 \mathrm{aR}$, is more potent than finasteride due to its ability to inhibit both type I and II isoenzymes, thus leading to a $90 \%$ reduction in DHT serum levels, whereas finasteride reduces only 70\% [7]. There is well-documented evidence that systemic dutasteride is more potent than finasteride [16, 17]. According to a recent systematic review and metaanalysis regarding efficacy and safety of dutasteride compared with finasteride, the 2 drugs appear to show similar rates of adverse reactions, especially in sexual dysfunction [8]. Dutasteride's adverse effects and its long half-life (4-5 weeks) are the main factors contributing to the reluctance on its oral use. Mesotherapy, a technique which involves microinjections of medications into the middle layer of the skin, could be a feasible alternative to avoid systemic exposure and unwanted side effects [18]. However, although there are some case series and small randomized clinical trials (RCT) using intralesional dutasteride, there is currently not enough evidence regarding its comparable efficacy to systemic administration. Therefore, we aimed to perform a systematic review in order to collect and analyze the scientific literature with the purpose of comparing the efficacy of both administration routes. Intralesional route would be an attractive alternative to avoid systemic effects if we could prove rather similar or superior efficacy as well as a better safety profile.

\section{Methods}

Study Design

This systematic review adheres to the Preferred Reporting Items for Systematic Reviews and Meta-Analyses Protocols (PRISMA-P) checklist. The present protocol was submitted to the International Prospective Register of Systematic Reviews (PROSPERO) under the number 153191.

\section{Eligibility Criteria}

Only RCT assessing the effect of either intralesional or oral dutasteride compared with a respective placebo, as well as RCT directly comparing the efficacy of oral versus intralesional dutasteride, were included. To be considered for inclusion, each study must have had a minimum follow-up of 8 weeks. There was no restriction regarding language, dose, and number of interventions in each group.

Subjects in each study were humans of any gender, with a minimum age of 18 years and a confirmed physician's diagnosis of AGA, independently of the use of previous pharmacological treatments; nevertheless, in this case, subjects must have undergone a washout period before the intervention. Studies which included subjects who had undergone previous invasive therapies for AGA treatment or subjects with an underlying pathology or treatment affecting hair growth were excluded from the review even though they had an AGA diagnosis. Crossover and quasi-experimental studies were also not considered for inclusion.

\section{Sources}

An experienced librarian performed a search strategy in the following databases: MEDLINE, Scopus, Web of Science, and EMBASE. The search ranged from each database's inception date until October 2019 by using a combination of keywords and $\mathrm{MeSH}$ terms regarding the population of study (AGA), the intervention being evaluated (oral and intralesional dutasteride), the main objective of our study (efficacy and operationalized as regrowth of hair), and the study designs considered for inclusion (RCT). An additional search was performed on previous systematic reviews on the topic to ensure not missing any possible studies.

\section{Study Selection}

After the search strategy was performed and duplicates were removed, 2 reviewers worked independently and in duplicate to screen the included references. The study selection process consisted of 2 phases, screening of titles and abstracts and full-text screening. In the 1 st phase, studies were either included or excluded for the full-text phase according to the information listed in their title or abstract, and any disagreement between reviewers was passed on to the next phase. In the 2 nd phase, all included references were analyzed in their fulltext form to decide final inclusion or exclusion, and any disagreement in this phase was resolved by either consensus or intervention of a 3 rd reviewer. A pilot study was performed before each phase to assure an adequate inter-rater agreement, defined as a Cohen's kappa coefficient over 0.7 . The study selection process was performed in the Distiller-SR software (Evidence Partners).

\section{Data Collection}

A web-based form was designed for the data collection process where 2 data extractors worked independently and in duplicate. The following information was collected: title of publication, Clin- 
Fig. 1. Flowchart of the number of studies identified and included in this systematic review.

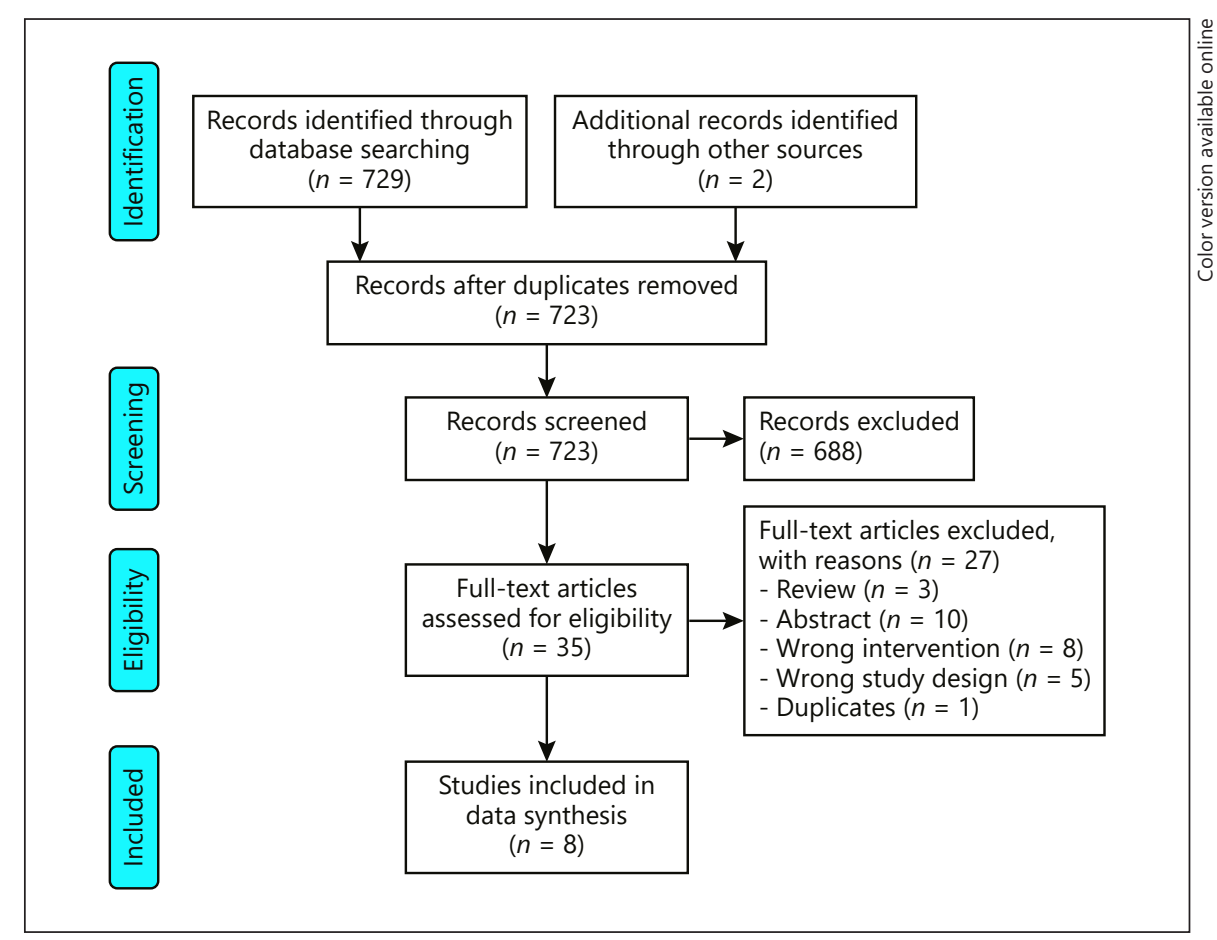

ical Trials registry when available, 1st author, year of publication, country of origin, study objectives and design, arms of treatment, administration route, dose, total dose received, frequency of intervention, study duration and follow-up, total number of randomized subjects, subjects that completed the study, demographic and baseline characteristics (age, gender, race, time of evolution, and Hamilton-Norwood classification), adverse events (reduced libido and erectile and ejaculatory dysfunction), quality of life, and selfassessments when available, as well as pull test, degree of DHT suppression, hair counts and diameter, and outcome and results. Also, possible conflicts of interest and finance resources were registered.

Risk of Bias in Individual Studies and Quality Assessment

A systematic assessment of the risk of bias in each included individual study was performed with the COCHRANE Risk of Bias 2.0 tool ( RoB 2.0) which covers the following domains: bias arising from the randomization process, bias due to deviations from intended interventions, bias due to missing outcome data, bias in measurement of the outcome, and bias in selection of the reported results.

We addressed the possibility of nonpublication and dissemination bias by performing an extensive literature search in Cochrane Central Register of Controlled Trials and ClinicalTrials.gov, as well as additional sites that exclusively address gray literature. Where possible, a funnel plot was performed to assess this situation.

\section{Statistical Analysis}

A meta-analysis was performed for oral dutasteride versus placebo and intralesional dutasteride versus placebo alone since none of the included studies directly compared oral versus intralesional dutasteride. Numerical variables were reported as mean and standard deviation, whereas categorical ones as frequency and percentage. Meta-analysis was performed using the inverse variance method, utilizing mean differences when comparing numerical outcomes (mean change in hair count) and odds ratios when comparing frequencies (proportion of adverse events) among groups. Heterogeneity was measured by using the $I^{2}$ statistic, and it was considered low when this value was less than $50 \%$, moderate when it was greater than $50 \%$ but less than $80 \%$, and high when higher than the latter value. When analysis had low heterogeneity, a fixedeffect model was used; on the contrary, a random-effects model was applied. Statistical analysis was performed under the program Review Manager V 5.3.

\section{Results}

\section{Study Selection}

Initially, the multiple database search identified 729 publications. Of these, 688 studies were excluded because they did not meet the inclusion criteria. Subsequently, 35 full-text articles were reviewed for possible eligibility and 27 were excluded for different reasons. Thus, the remaining 9 clinical trials were carefully reviewed, and 1 study was removed from the data synthesis due to duplication. Finally, 8 clinical trials met the inclusion criteria and were selected for data synthesis. The complete study selection process is shown in Figure 1. 


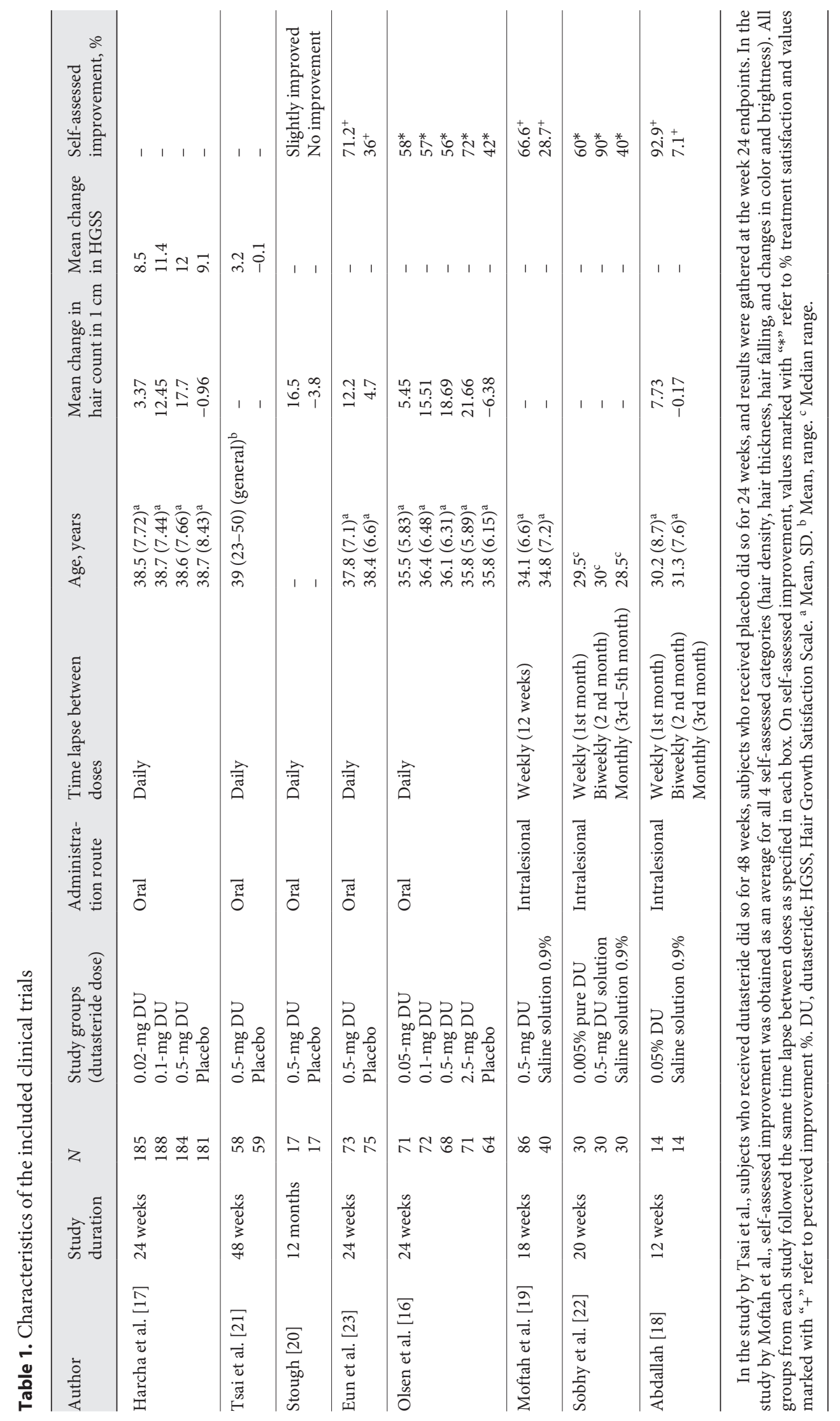




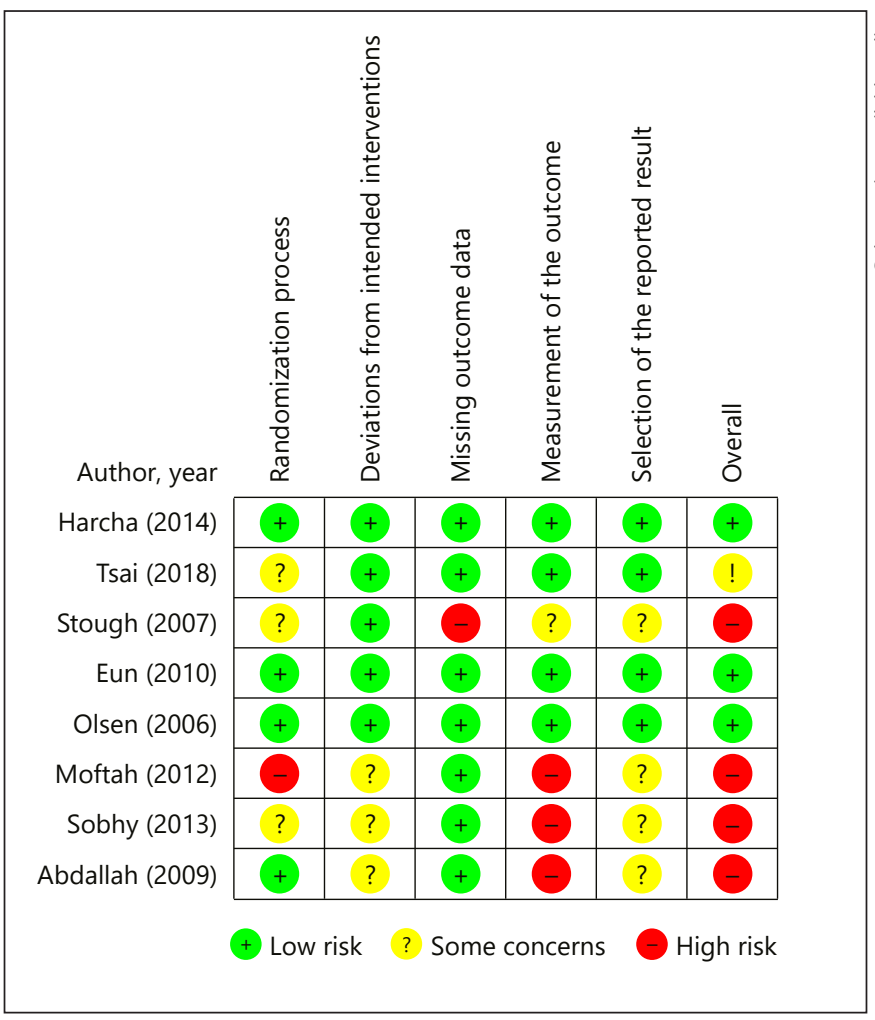

Fig. 2. Risk of bias assessment of the included studies according to the Cochrane guidelines.

\section{Selection and Characteristics of Included Studies}

Selected studies comprised a total of 1,627 patients, including 480 participants in the placebo group and 1,147 in the active treatment groups. Included RCT were published between 2006 and 2018. Of the 1,627 patients included in the analysis, 1,487 were men. Out of the 1,627 patients included in the analysis, 987 were receiving oral dutasteride and 160 intralesional administration. The range of the treatment period was from 12 to 48 weeks. Characteristics of the included clinical trials are shown in Table 1.

\section{Risk of Bias Assessment}

According to the Cochrane RoB 2.0 criteria, 1 study was ranked having a high risk of bias [19] and 3 having some concerns of bias [20-22] concerning the randomization process. Subsequently, 3 other studies were ranked as having some concerns of bias $[18,19,22]$ in the deviations from intended intervention domain. Regarding missing outcome data, 1 trial exhibited high risk of bias [20]. With respect to the measurement of the outcome criteria, 3 studies were ranked as having high risk of bias
$[18,19,22]$ and 1 study having some concerns [20]. In addition, 4 studies revealed some concerns in the selection of the reported results [18-20, 22]. Finally, 4 studies showed high risk of bias [18-20,22], 1 had some concerns [20] for overall bias, and 3 were ranked as having a low risk of bias $[16,17,23]$. The complete risk of bias assessment is shown in Figure 2.

\section{Effect of Treatment with Dutasteride (Oral or Intralesional) on Hair Count}

Based on the data available from the included studies, we compared the mean change in hair count between the intervention (oral or intralesional dutasteride) and its respective control group (placebo) [1623]. All studies had a minimum intervention of 12 weeks of treatment. After a treatment of at least 24 weeks with oral dutasteride, a significant increase in the mean of the hair count was observed in patients receiving oral dutasteride over placebo (MD: 15.92 hairs [95\% CI: 9.87-21.96]; $\left.p=<0.00001 ; I^{2}=90 \%\right)[16,17,20,21$, 23]. Regarding intralesional dutasteride, only 1 study [18] reported the mean change in hair count and therefore a meta-analysis was not possible; however, a statistically significant difference benefiting the intervention was observed in the mean change of hair count when comparing it to placebo (MD: 7.90 hairs [95\% CI: 7.148.66]; $p=<0.00001$ ) (shown in Figure 3).

\section{Effect of Dutasteride Treatment and Adverse Events Libido}

Libido was one of the adverse events that was reported in at least 3 out of 5 studies evaluating oral dutasteride. When compared to placebo, after a treatment of at least 24 weeks with oral dutasteride, a nonsignificant increase in the proportion of decreased sexual desire in the dutasteride group was found (OR: 2.03 [95\% CI: 0.77-5.34]; $p=0.15 ; I^{2}=0 \%$ ) (shown in Fig. 4 a). No study with intralesional treatment reported libido adverse events.

\section{Erectile Dysfunction}

Erectile dysfunction was reported in 4 out of the 5 studies evaluating oral dutasteride where, after a treatment of at least 24 weeks, a nonsignificant increase in the proportion of erectile dysfunction in the dutasteride group was found when comparing it to placebo (OR: 0.67 [95\% CI: $0.36-1.25] ; p=0.21 ; I^{2}=41 \%$ ) (Fig. 4 b). No study with intralesional treatment reported erectile dysfunction adverse events. 


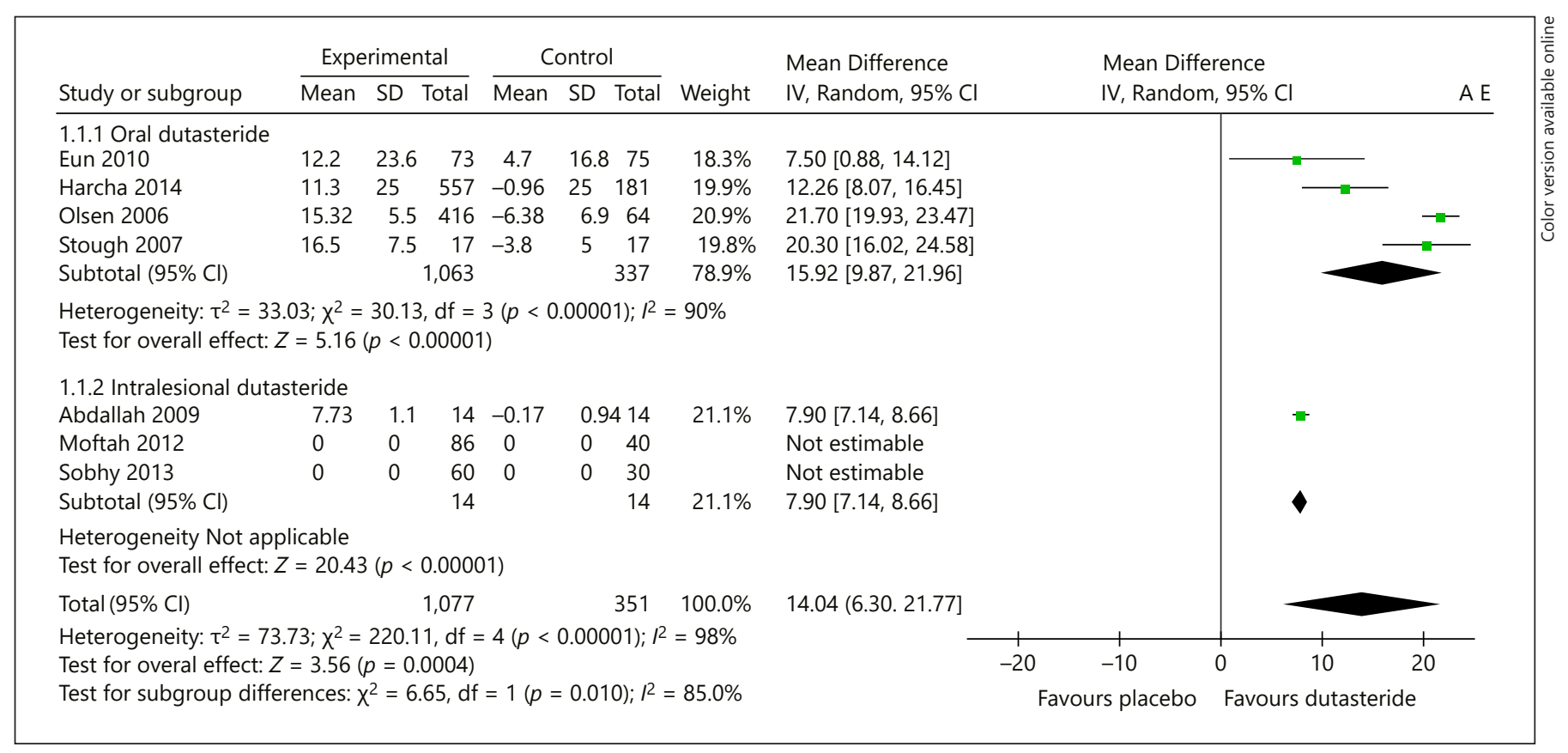

Fig. 3. Forest plot displaying the mean difference and $95 \%$ confidence intervals for the hair count at pre- and post-dutasteride treatment (oral and intralesional).

Ejaculatory Dysfunction

Ejaculation dysfunction was reported in 4 out of the 5 studies evaluating oral dutasteride, where after a treatment of at least 24 weeks with oral dutasteride, a nonsignificant increase in the proportion of ejaculation dysfunction was found when comparing it to placebo (OR: 0.96 [95\% CI: $0.44-2.11$ ]; $p=0.92 ; I^{2}=0 \%$ ) (Fig. 4c). No study with intralesional treatment reported ejaculation dysfunction adverse events.

\section{Discussion}

AGA is characterized by progressive hair loss with age, eventually leading to other psychological effects related to self-appreciation. DHT is believed to be the main pathogenic factor in AGA, which results in miniaturization of hair follicles and conversion of terminal hairs into vellus hairs [8]. By inhibiting $5 \mathrm{aR}$ activity, conversion of testosterone to DHT can be diminished, in order to treat AGA.

After a detailed literature search, no study directly comparing the effects of oral and intralesional dutasteride could be encountered. Five clinical trials using an oral route and 3 an intralesional route, in comparison with placebo, were included for this systematic review. Three of the 5 studies evaluating oral dutasteride were classified as having low risk of bias; however, all 3 studies evaluating intralesional dutasteride were classified as having high risk.

Apparently mean change in hair count from baseline in $\mathrm{cm}^{2}$ was greater with oral over intralesional dutasteride. Our pooled analysis of 4 studies evaluating oral dutasteride estimated a mean change in hair growth of 15.92 hairs per $\mathrm{cm}^{2}$, when indirectly compared to a mean change of 7.9 hairs per $\mathrm{cm}^{2}$ in the 1 study of intralesional dutasteride that reported this outcome. A clear difference favoring oral dutasteride could be observed; however, it was not possible to perform a statistical comparison between both treatments.

Regarding the presence of sexual adverse events in studies evaluating oral dutasteride against placebo, our pooled analysis showed no difference in the proportion of these outcomes. These results are consistent with a recent meta-analysis performed on the subject [24].

With respect to intralesional therapy with dutasteride, some considerations should be highlighted. There are no reports regarding sexual side effects in the medical literature; thus, this does not appear to be a serious concern [13]. Furthermore, some other side effects such as patchy hair loss, cicatricial alopecia, and multifocal scalp abscess described in the medical literature are merely based on case reports [7]. 


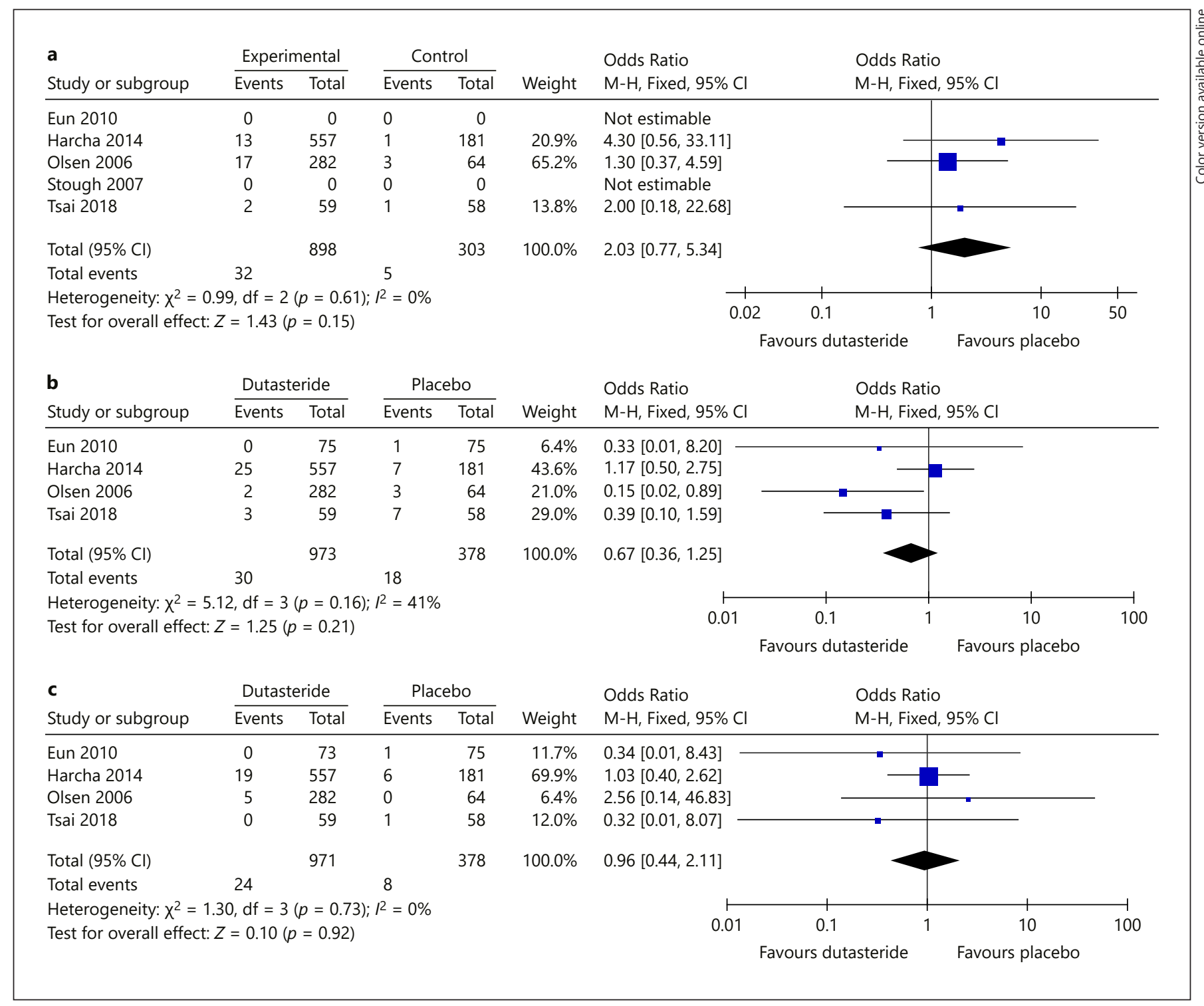

Fig. 4. a Forest plot displaying the difference in proportions and 95\% confidence intervals for the libido adverse events at pre- and post-oral dutasteride treatment. b Forest plot displaying the difference in proportions and $95 \%$ confidence intervals for the erectile

Also to consider is the fact that currently there is no standardized intervention protocol for intralesional dutasteride. Future studies should focus on establishing the optimal number of treatments and dosages, frequency, administration techniques, and overall safety.

Our study is limited by the fact that direct comparisons could not be performed since there are no published clinical trials comparing the efficacy of oral versus intralesional dutasteride; therefore, our conclusions on the subject are not statistically based. Besides, half of the studies dysfunction adverse events at pre- and post-oral dutasteride treatment. c Forest plot displaying the difference in proportions and $95 \%$ confidence intervals for the ejaculation dysfunction adverse events at pre- and post-oral dutasteride treatment.

included for this systematic review were classified as having "high risk of bias," limiting the credibility of the results.

\section{Conclusion}

To date, the use of oral dutasteride seems to be the optimal treatment modality for AGA since it appears to be more effective than intralesional dutasteride. Side effects 
of systemic dutasteride have been widely studied and reported, making physicians and patients aware of potential adverse events. Intralesional route can be a feasible alternative for those unwilling to receive systemic treatment, avoiding the well-known systemic effects. Nevertheless, until more clinical trials evaluating the efficacy and safety of intralesional dutasteride are published, physicians should be cautious before recommending it as a better option over oral administration.

\section{Statement of Ethics}

This systematic review adheres to the Preferred Reporting Items for Systematic Reviews and Meta-Analyses Protocols (PRISMA-P) checklist and was submitted to the International Prospective Register of Systematic Reviews (PROSPERO).

\section{Conflict of Interest Statement}

There are no conflicts of interest.

\section{Funding Sources}

The authors did not receive any funding.

\section{Author Contributions}

M.H. and R.G. conceived the idea. N.A., H.L., and J.M. verified the analytical methods. M.H. wrote the manuscript. S.O. and M.G. contributed to the final version of the manuscript.

\section{References}

1 Blume-Peytavi U, Blumeyer A, Tosti A, Finner A, Marmol V, Trakatelli M, et al. S1 guideline for diagnostic evaluation in androgenetic alopecia in men, women and adolescents. Br J Dermatol. 2011 Jan;164(1):5-15.

2 Hamilton JB. Patterned loss of hair in man; pathogenesis and prognosis. Ann N Y Acad Sci. 1951 Mar;53(3):729-34.

3 Gan DC, Sinclair RD. Prevalence of male and female pattern hair loss in Maryborough. J Investig Dermatol Symp Proc. 2005 Dec;10(3): 184-9.

4 Randall VA. Androgens and human hair growth. Clin Endocrinol. 1994 Apr;40(4): 439-57.

5 Ellis JA, Harrap SB. The genetics of androgenetic alopecia. Clin Dermatol. 2001 Mar-Apr; 19(2):149-54.

6 Mahé YF, Michelet JF, Billoni N, Jarrousse F, Buan B, Commo S, et al. Androgenetic alopecia and microinflammation. Int J Dermatol. 2000 Aug;39(8):576-84.

7 Busanello EB, Turcatel E. Androgenic alopecia and dutasteride in hair mesotherapy: a short review. Our Dermatol Online. 2017 Sep; 9(1):75-9.

8 Zhongbao Z, Shiqiang S, Zhenli G, Jitao W, Jiajia M, Yuanshan C. The efficacy and safety of dutasteride compared with finasteride in treating men with androgenetic alopecia: a systematic review and meta-analysis. Clin Interv Aging. 2019 Feb;14:399-406.

9 Kaufman KD. Androgen metabolism as it affects hair growth in androgenetic alopecia. Dermatol Clin. 1996 Oct;14(4):697-711.

10 Sato T, Sonoda T, Itami S, Takayasu S. Predominance of type I $5 \alpha$-reductase in apocrine sweat glands of patients with excessive or abnormal odour derived from apocrine sweat (osmidrosis). Br J Dermatol. 1998 Nov; 139(5):806-10.

11 Thiboutot D, Harris G, Iles V, Cimis G, Gilliland K, Hagari S. Activity of the type 15 alpha-reductase exhibits regional differences in isolated sebaceous glands and whole skin. J Invest Dermatol. 1995 Aug;105(2):209-14.

12 Chen W, Zouboulis C, Orfanos CE. The 5-alpha-reductase system and its inhibitors. Recent development and its perspective in treating androgen-dependent skin desorders. Dermatology. 1995 Dec;193(3):177-84.

13 Tsunemi Y, Irisawa R, Yoshiie H, Brotherton $B$, Ito $H$, Tsuboi R, et al. Long-term safety and efficacy of dutasteride in the treatment of male patients with androgenetic alopecia. J Dermatol. 2016 Sep;43(9):1051-8.

14 Rogers NE, Avram MR. Medical treatments for male and female pattern hair loss. J Am Acad Dermatol. 2008 Oct;59(4):547-8.

15 Kanti V, Messenger A, Dobos G, Reygagne P, Finner A, Blumeyer A, et al. Evidence-based (S3) guideline for the treatment of androgenetic alopecia in women and in men: short version. J Eur Acad Dermatol Venereol. 2018 Jan;32(1):11-22.

16 Olsen EA, Hordinsky M, Whiting D, Stough $D$. The importance of dual 5 a -reductase inhibition in the treatment of male pattern hair loss: results of a randomized placebo-controlled study of dutasteride versus finasteride. J Am Acad Dermatol. 2006 Dec;55(6):101423.

17 Gubelin Harcha W, Barboza Martínez J, Tsai TF, Katsuoka K, Kawashima M, Tsuboi R, et al. A randomized, active- and placebo-controlled study of the efficacy and safety of different doses of dutasteride versus placebo and finasteride in the treatment of male subjects with androgenetic alopecia. J Am Acad Dermatol. 2014 Mar;70(3):489-e3.

18 Abdallah M. Mesotherapy using dutasteridecontaining solution in male pattern hair loss: a controlled pilot study. J Pan-Arab League Dermatol. 2009 Feb;20(1):137-45.

19 Moftah N, Moftah N, Ahmed N, Hamed Y, Ghannam B, Ibrahim M. Mesotherapy using dutasteride-containing preparation in treatment of female pattern hair loss : photographic, morphometric and ultrustructural evaluation. J Eur Acad Dermatol Venereol. 2013 Jun;27(6):686-93.

20 Stough D. Dutasteride improves male pattern hair loss in a randomized study in identical twins. J Cosmet Dermatol. 2007 Mar;6(1):913.

21 Tsai TF, Choi GS, Kim BJ, Kim MB, Ng CF, Kochhar $\mathrm{P}$, et al. Prospective randomized study of sexual function in men taking dutasteride for the treatment of androgenetic alopecia. J Dermatol. 2018 Jul;45(7):799-804.

22 Sobhy N, Aly H, El Shafee A, El Deeb M. Evaluation of the effect of injection of dutasteride as mesotherapeutic tool in treatment of androgenetic alopecia in males. Our Dermatol Online. 2013;4(1):40-5.

23 Eun HC, Kwon OS, Yeon JH, Shin HS, Kim BY, Ro BI, et al. Efficacy, safety, and tolerability of dutasteride $0.5 \mathrm{mg}$ once daily in male patients with male pattern hair loss: a randomized, double-blind, placebo-controlled, phase III study. J Am Acad Dermatol. 2010 Aug;63(2):252-8.

24 Liu L, Zhao S, Li F, Li E, Kang R, Luo L, et al. Effect of $5 \alpha$-reductase inhibitors on sexual function: a meta-analysis and systematic review of randomized controlled trials. J Sex Med. 2016 Sep;13(9):1297-310. 